\title{
Topological Function Spaces
}

\author{
by
}

\author{
Alycia M. Kolat
}

Submitted in Partial Fulfillment of the Requirements

for the Degree of

Masters

in the

Mathematics

Program

YOUNGSTOWN STATE UNIVERSITY

May, 2011

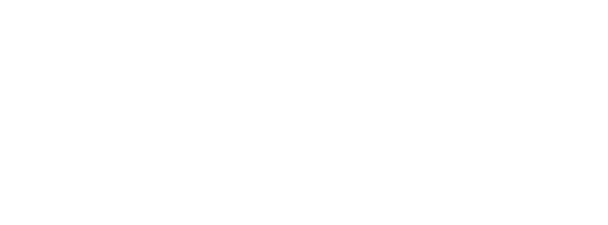




\section{Topological Function Spaces}

Alycia M. Kolat

I hereby release this thesis to the public. I understand that this thesis will be made available from the OhioLINK ETD Center and the Maag Library Circulation Desk for public access. I also authorize the University or other individuals to make copies of this thesis as needed for scholarly research.

Signature:

Alycia M. Kolat, Student

Date

Approvals:

Dr. Jamal Tartir, Thesis Advisor

Date

Dr. Stephen Rodabaugh, Committee Member

Date

Dr. Eric Wingler, Committee Member

Date

Peter J. Kasvinsky, Dean of School of Graduate Studies \& Research Date 
(C)

Alycia M. Kolat

2011 


\begin{abstract}
This thesis is an introduction to function spaces endowed with the topology of pointwise convergence, abbreviated $C_{p}$-theory, and a look at a small portion of the open questions in the field. In the introductory chapters, several different cardinal functions are presented and then used to characterize properties of $C_{p}$-spaces. The final chapters explore several open questions pertaining to Lindelöfness and metacompactness of $C_{p}$-spaces.
\end{abstract}




\section{Contents}

$\begin{array}{ll}\text { Index } & 1\end{array}$

1 Basics 1

1.1 History . . . . . . . . . . . . . . . . . . . . . . 1

1.2 Terminology . . . . . . . . . . . . . . . . . . . . . 1

1.3 Cardinal Invariants . . . . . . . . . . . . . . . . . . . . . . . . . . . . . .

1.4 Compactness Properties . . . . . . . . . . . . . . 8

2 Introduction to $C_{p}(X) \quad 10$

2.1 Definitions . . . . . . . . . . . . . . . . . 10

2.2 Properties . . . . . . . . . . . . . . . . . . . . . . . . . . . . . . . . . . . . .

2.3 Examples . . . . . . . . . . . . . . . . . . . 14

$3 C_{p}(X)$ and Lindelöfness $\quad 18$

4 Lindelöfness of $C_{p}(X)$ Examples $\quad 20$

$4.1 X$ has a Single Non-Isolated Point . . . . . . . . . . . . . . . . 20

$4.2 X$ is a Michael Space . . . . . . . . . . . . . . . . . . 21

$4.3 X$ is a Mrówka Space . . . . . . . . . . . . . . . . . . . 22

$5 C_{p}(X)$ and Metacompactness $\quad 24$

$\begin{array}{lr}\text { References } & 26\end{array}$ 


\section{Chapter 1}

\section{Basics}

This thesis focuses on function spaces with the topology of pointwise convergence or $C_{p}$-theory for short. The beginning sections provide a brief recollection of concepts from general topology needed to begin studying $C_{p}$-theory. The second chapter provides a basic understanding of the properties of a space $C_{p}(X)$. The final three chapters of the thesis consider open problems in $C_{p}$-theory and explore partial solutions.

\section{$1.1 \quad$ History}

The study of sets, or spaces, of functions began with the works of Ascoli, Arzelà and Hadamard. Their work proposed questions that helped to shape the work of mathematicians such as Fréchet, Riesz, Weyl and Hausdorff.

While the contributions of all of the above mentioned mathematicians have helped develop the field of functional analysis, it was Tychonoff who, in 1935 with Über einen Funktionenraum [12], first began placing a topology on spaces of functions. Merging together ideas from general topology, algebraic topology, and functional analysis, mathematicians began studying $C_{p}$-theory [13].

Without question the first mathematician to realize the significance of studying $C_{p}$-theory for its own sake was Alexander Arhangel'skii. Arhangel'skii was the first to propose open questions and wrote one of the few books [1] devoted entirely to the subject.

Since then, many mathematicians have studied $C_{p}$-theory and made significant contributions. However, a great deal of $C_{p}$-theory remains new and it is difficult to avoid finding open questions.

\subsection{Terminology}

In this section, terminology and concepts will be introduced that play an important role in studying and understanding $C_{p}$-theory. We begin by recalling some separation 
axioms, as all spaces throughout this thesis will be considered Tychonoff. Why this is the case will be described more in the next chapter.

Definition 1.2.1. A topological space $X$ is completely regular if whenever $E \subseteq X$ is closed and $x \notin E$, there exists a continuous function $f: X \rightarrow[0,1]$ such that $f(x)=0$ and $f(y)=1$ for all $y \in E$. That is, $E \subseteq f^{-1}(1)$.

Definition 1.2.2. A completely regular $T_{1}$-space is called Tychonoff or $T_{3 \frac{1}{2}}$.

Definition 1.2.3. A topological space $X$ is normal if whenever $E$ and $F$ are disjoint, closed sets, there are disjoint open sets, $U$ and $V$, with $E \subseteq U$ and $F \subseteq V$.

Definition 1.2.4. A space is called Lindelöf if every open cover has a countable subcover.

The following definitions will be seen throughout later chapters to describe properties of spaces. These properties are used to show results towards solving some open problems in the theory of topological function spaces.

Definition 1.2.5. Let $(X, \mathcal{T})$ be a topological space. Then $\mathcal{B}$ is a base for $\mathcal{T}$ if for all $U \subseteq \mathcal{T}$, there exists $\mathcal{B}^{\prime} \subseteq \mathcal{B}$ such that $U=\cup \mathcal{B}^{\prime}$

Definition 1.2.6. Let $(X, \mathcal{T})$ be a topological space and $x \in X$. Then $\mathcal{B} \subseteq \mathcal{T}$ is called a local base at $x$ if

i. $x \in B$ for all $B \in \mathcal{B}$; and

ii. whenever $x \in U \in \mathcal{T}$, there exists $B \in \mathcal{B}$ such that $x \in B \subseteq U$.

Definition 1.2.7. A topological space with a countable base is called second countable.

Definition 1.2.8. A topological space $X$ is first countable if for every $x \in X$, there is a countable local base at $x$.

Definition 1.2.9. A topological space is separable if it has a countable dense subset. 
Definition 1.2.10. A topological space $(X, \mathcal{T})$ is metrizable if there is a metric $d$ on $X$ such that $\mathcal{T}=\mathcal{T}_{d}$.

Definition 1.2.11. A topological space $X$ is scattered if for every closed subset $C$ of $X$, the set of isolated points of $C$ is dense in $C$.

Definition 1.2.12. A subset of a topological space is $G_{\delta}$ if it is the intersection of a countable collection of open sets.

Definition 1.2.13. A subset of a topological space is $F_{\sigma}$ if it is the union of a countable collection of closed sets.

This last set of definitions will briefly remind the reader of the product topology by first recalling the topology on $X \times Y$, then introducing infinite products.

Definition 1.2.14. Let $\left(X, \mathcal{T}_{X}\right)$ and $\left(Y, \mathcal{T}_{Y}\right)$ be topological spaces. Then $\{U \times V$ : $\left.U \in \mathcal{T}_{X}, V \in \mathcal{T}_{Y}\right\}$ is a base for $X \times Y$ and generates a topology called the product topology on $X \times Y$.

Definition 1.2.15. Suppose $\Lambda$ is a set and $X_{\alpha}$ is a set for all $\alpha \in \Lambda$. Then

$$
\prod_{\alpha \in \Lambda} X_{\alpha}=\left\{f: \Lambda \rightarrow \bigcup_{\alpha \in \Lambda} X_{\alpha} \mid f(\alpha) \in X_{\alpha} \text { for all } \alpha \in \Lambda\right\} .
$$

Definition 1.2.16. A basic open set in $\Pi_{\alpha \in \Lambda} X_{\alpha}$ is of the form $\prod_{\alpha \in \Lambda} U_{\alpha}$ where each $U_{\alpha}$ is open in $X_{\alpha}$ and $U_{\alpha}=X_{\alpha}$ for all but finitely many $\alpha$. The topology on $\Pi_{\alpha \in \Lambda} X_{\alpha}$ is the topology generated by this base.

\subsection{Cardinal Invariants}

The next definitions are an introduction to cardinal invariants. These will be used in the same manner as the previous definitions to describe various spaces in theorems in later chapters. Cardinal invariants are useful in many proofs and provide some different properties than the definitions given in the previous section. The first cardinal invariants that will be seen are useful for describing a topological space as a whole. 
Definition 1.3.1. The Lindelöf number is the cardinal

$$
l(X)=\min \{\kappa: \text { every open cover of } X \text { has a subcover of power } \leq \kappa\} .
$$

Definition 1.3.2. Let $(X, \mathcal{T})$ be a topological space. The Suslin number of $X$ is the cardinal

$$
c(X)=\sup \{|\mathcal{U}|: \mathcal{U} \subseteq \mathcal{T} \text { is disjoint }\}
$$

Definition 1.3.3. The weight of a space $X$ is the cardinal

$$
w(X)=\min \{|\mathcal{B}|: \mathcal{B} \text { is a base of } X\} .
$$

Since the weight is the minimal cardinality of a base of $X$, if $w(X)=\omega$ then the space $X$ is second countable.

Definition 1.3.4. A network in a space $(X, \mathcal{T})$ is a family $\mathcal{N}$ of subsets of the set $X$ such that for any open $U \in \mathcal{T}$, there is $\mathcal{N}^{\prime} \subseteq \mathcal{N}$ with $\cup \mathcal{N}^{\prime}=U$.

Definition 1.3.5. The network weight is the cardinal

$$
n w(X)=\min \{|\mathcal{N}|: \mathcal{N} \text { is a network of } X\} .
$$

Definition 1.3.6. A condensation is a bijective continuous map.

Definition 1.3.7. The $i$-weight of $X$ is the cardinal

$$
i w(X)=\min \{|\kappa|: \text { there is a condensation of } X \text { onto a space of weight }<\kappa\} .
$$

Definition 1.3.8. The density of a space $X$ is the cardinal

$$
d(X)=\min \{|A|: A \text { is dense in } X\} .
$$

A space $X$ is separable if $d(X)=\omega$.

Definition 1.3.9. The spread of a space $X$ is the cardinal

$$
s(X)=\sup \{|D|: D \text { is a discrete subset of } X\} .
$$


Definition 1.3.10. The extent of a space $X$ is the cardinal

$$
\operatorname{ext}(X)=\sup \{|D|: D \subseteq X \text { is both closed and discrete }\}
$$

The next set of cardinal invariants helps to describe topological spaces at a given point. These are sometimes referred to as point cardinal invariants.

Definition 1.3.11. Let $\kappa$ be an infinite cardinal number and $A \subseteq X$. Let $[A]_{\kappa}=$ $\cup\{\bar{B}: B \subseteq A$ and $|B| \leq \kappa\}$. Then the tightness of $X$ is defined as

$$
t(X)=\min \left\{\kappa: \bar{A}=[A]_{\kappa} \text { for every } A \subseteq X\right\} .
$$

Definition 1.3.12. Let $(X, \mathcal{T})$ be a topological space, $A \subseteq X$ and

$$
\psi(A, X)=\min \{|\mathcal{U}|: \mathcal{U} \subseteq \mathcal{T} \text { and } \cap \mathcal{U}=A\} .
$$

Then $\psi(A, X)$ is the pseudocharacter of $A$ in $X$. If the set $A=\{x\}$, we use the notation $\psi(x, X)$.

Notice that $G_{\delta}$-sets have a countable pseudocharacter.

Definition 1.3.13. The cardinal $\psi(X)=\sup \{\psi(x, X): x \in X\}$ and is called the pseudocharacter of $X$.

Definition 1.3.14. Let $(X, \mathcal{T})$ be a topological space and $A \subseteq X$. Then a family $\mathcal{B} \subseteq \mathcal{T}$ is an outer base of $A$ in $X$ if $\mathcal{B} \neq \emptyset, A \subseteq \cap \mathcal{B}$, and for every $U \in \mathcal{T}$ with $A \subseteq U$, there is $V \in \mathcal{B}$ such that $V \subseteq U$.

Notice that if the subset $A$ is a singleton, then an outer base is a local base.

Definition 1.3.15. Let $X$ be a topological space and $A \subseteq X$. The minimum cardinality of the outer bases of $A$ in $X$ is called the character of $A$ in $X$ and is denoted by $\chi(A, X)$. If $A=\{x\}$, the notation $\chi(x, X)$ is used.

Definition 1.3.16. The cardinal $\chi(X)=\sup \{\chi(x, X): x \in X\}$ and is called the character of $X$. 
Definition 1.3.17. Let $X$ be a space. Then the set $\Delta_{X}=\{(x, x): x \in X\} \subseteq X \times X$ is called the diagonal of $X$. The cardinal $\Delta(X)=\psi\left(\Delta_{X}, X \times X\right)$ is called the diagonal number of $X$.

Now that several cardinal invariants have been defined, it will be useful to see the relationships between them. The following lemmas and theorem illustrate some of the most basic relationships between the previously defined cardinal invariants. The proofs in this section are adapted from [9].

Lemma 1.3.18. If a space $X$ is second countable and $A$ is an infinite set, then for any $Y \subseteq X^{A}$, we have that $w(Y) \leq|A|$.

Proof. Fix a countable base $\mathcal{B}$ in $X$ and let $a_{1}, \ldots, a_{n} \in A$ and $O_{1}, \ldots, O_{n} \in \mathcal{B}$ be given. Let $\left[a_{1}, \ldots, a_{n} ; O_{1}, \ldots, O_{n}\right]=\left\{x \in X^{A}: x\left(a_{i}\right) \in O_{i}\right.$ for all $\left.i \leq n\right\}$. Then the family $\mathcal{C}=\left\{\left[a_{1}, \ldots, a_{n} ; O_{1}, \ldots, O_{n}\right]: n \in \mathbb{N}, a_{i} \in A\right.$ and $O_{i} \in \mathcal{B}$ for all $\left.i \leq n\right\}$ is a base for $X^{A}$. Also, $|\mathcal{C}| \leq|A|$ which implies that $w\left(X^{A}\right) \leq|A|$. Now let $\mathcal{C}^{\prime}=\{U \cap Y$ : $U \in \mathcal{C}\}$. Then $\mathcal{C}^{\prime}$ forms a base for $Y$ and $\left|\mathcal{C}^{\prime}\right| \leq|\mathcal{C}| \leq A$. Thus $w(Y) \leq A$.

Lemma 1.3.19. There exists a set $A \subseteq C(X,[0,1])$ with $|A| \leq n w(C(X,[0,1]))=\kappa$ such that, for any distinct $x, y \in X$, there is $f \in A$ for which $f(x) \neq f(y)$.

Proof. Let $\mathcal{N}$ be a network of $X$. Define a pair $p=\left(N_{1}, N_{2}\right) \in \mathcal{N} \times \mathcal{N}$ as being marked if there is a function $f_{p} \in C(X,[0,1])$ such that $f\left(N_{1}\right) \subseteq\left[0, \frac{1}{3}\right]$ and $f\left(N_{2}\right) \subseteq$ $\left[\frac{2}{3}, 1\right]$. Let $A=\left\{f_{p}: p\right.$ is a marked pair $\}$. Now by construction, we have that $|A| \leq$ $|\mathcal{N} \times \mathcal{N}| \leq \kappa$. Let $x, y \in X$ such that $x \neq y$. Since $X$ is Tychonoff (and thus completely regular), there is $g \in C(X,[0,1])$ such that $g(x)=0$ and $g(y)=1$. Let $U=g^{-1}\left(\left[0, \frac{1}{3}\right)\right)$ and $V=g^{-1}\left(\left(\frac{2}{3}, 1\right]\right)$. Then $x \in U \in \mathcal{T}_{X}$ and $y \in V \in \mathcal{T}_{X}$. Since $\mathcal{N}$ is a network, this implies there exist $N_{1}, N_{2} \in \mathcal{N}$ such that $x \in N_{1} \in U$ and $y \in N_{2} \in$ $V$. Now $g\left(N_{1}\right) \subseteq\left[0, \frac{1}{3}\right]$ and $g\left(N_{2}\right) \subseteq\left[\frac{2}{3}, 1\right]$. Thus $p=\left(N_{1}, N_{2}\right)$ is a marked pair and so $f_{p}=g \in A$. Hence, $f_{p}(x) \leq \frac{1}{3}$ and $f_{p}(y) \geq \frac{2}{3}$ and so $f_{p}(x) \neq f_{p}(y)$.

Theorem 1.3.20. For any space $X$,

1. $c(X) \leq d(X) \leq n w(X) \leq w(X)$;

2. $c(X) \leq s(X)$ and $\operatorname{ext}(X) \leq l(X) \leq n w(X) ;$

3. $\psi(X) \leq \chi(X)$ and $\psi(X) \leq i w(X) \leq n w(X)$;

4. $t(X) \leq \chi(X) \leq w(X)$ and $t(X) \leq n w(X)$. 
Proof. (1) Let $(X, \mathcal{T})$ be a topological space. Let $D \subseteq X$ be dense and let $\mathcal{U} \subset \mathcal{T}$ be a nonempty disjoint family. Choose $y(U) \in U \cap D$ for any $U \in \mathcal{U}$. Consider the mapping from $U \rightarrow y(U)$. Since $\mathcal{U}$ is pairwise disjoint, this is a one-to-one correspondence from $\mathcal{U}$ to $D$. Hence, $c(X) \leq d(X)$. Now let $\mathcal{N}$ be a network of $X$. Choose $x(N) \in N$ for some $N \in \mathcal{N}$ and consider $\{x(N): N \in \mathcal{N}\}$. Clearly, this set is dense in $X$. Thus, $d(X) \leq n w(X)$. Finally, by definition a base is also a network resulting in $n w(X) \leq w(X)$.

(2) Let $\mathcal{U} \subseteq \mathcal{T}$ be a disjoint family and choose $y(U) \in U$ for each $U \in \mathcal{U}$. Then the subspace $D=\{y(U): U \in \mathcal{U}\}$ is discrete and $|D|=|\mathcal{U}|$. Thus $c(X) \leq s(X)$.

Now, assume $l(X) \leq \kappa$ and let $D \subseteq X$ be closed and discrete. For each $d \in D$, choose $U_{d} \in \mathcal{T}$ such that $d \in U_{d}$ with $U_{d} \cap D=\{d\}$. Let $\mathcal{U}=\left\{U_{d}: d \in\right.$ $D\} \cup\{X \backslash D\}$. Clearly, $\mathcal{U}$ is an open cover of $X$. Since any subcover $\mathcal{V}$ of $X$ would need to contain $\left\{U_{d}: d \in D\right\}$, we see that $|\mathcal{V}|=|\mathcal{U}|$. Thus, by our assumption that $l(X) \leq \kappa$, we see that $|D|=|\mathcal{U}| \leq \kappa$, and so $\operatorname{ext}(X) \leq l(X)$. Finally, let $\mathcal{N}$ be a network of $X,|\mathcal{N}|=\kappa$ and $\mathcal{U} \subseteq \mathcal{T}$ be an open cover of $X$. Let $\mathcal{N}^{\prime}=\{N \in \mathcal{N}$ : there exists $U(N) \in \mathcal{U}$ with $N \subseteq U(N)\}$. Then $\mathcal{V}=\left\{U(N): N \in \mathcal{N}^{\prime}\right\}$ is a subcover of $\mathcal{U}$ with cardinality $<\kappa$. To see that $\mathcal{V}$ is a cover of $X$, let $x \in X$. Then there is $U \in \mathcal{U}$ with $x \in U$ and, since $\mathcal{N}$ is a network, there is $N \in \mathcal{N}$ with $x \in N \subseteq U$. But this implies that $N \in \mathcal{N}^{\prime}$. Thus, $x \in N \subseteq U(N)$, and so $\mathcal{V}$ is a cover of $X$. Hence, $l(X) \leq n w(X)$.

(3) Let $\mathcal{B}$ be a local base at a point $x \in X$. Then since $X$ is a Tychonoff space, for any $y \neq x, X \backslash\{y\}$ is an open neighborhood of $x$. Thus there is $U \in \mathcal{B}$ such that $x \in U \subseteq X \backslash\{y\}$ and so, $\cap \mathcal{B}=\{x\}$ which gives $\psi(X) \leq \chi(X)$.

Let $X, Y$ be topological spaces and $f: X \rightarrow Y$ be a condensation such that $w(Y) \leq \kappa$. Then by definition and previously proven properties, we have $\psi(Y) \leq$ $\chi(Y) \leq w(Y) \leq \kappa$. Now, for $x \in X$, let $y=f(x)$ and fix a family $\mathcal{V} \subseteq \mathcal{T}_{Y}$ such that $\cap \mathcal{V}=\{y\}$. Let $\mathcal{V}^{\prime}=\left\{f^{-1}(U): U \in \mathcal{V}\right\}$. Then $\mathcal{V}^{\prime} \subseteq \mathcal{T}_{X}$ and $\cap \mathcal{V}^{\prime}=\{x\}$. Hence, $\psi(X) \leq i w(X)$.

Let $\mathcal{N}$ be a network of $X$ such that $|\mathcal{N}|=n w(X)=\kappa$. Since $X$ is regular, we see that for $N \in \mathcal{N}$ we can take the closure of each $N$ and $\{\bar{N}: N \in \mathcal{N}\}$ is a network as well. Thus, we can consider all the elements of $\mathcal{N}$ to be closed. Now, let $A \subseteq C(X,[0,1])$ with $|A| \leq \kappa$. Let $\phi: X \rightarrow[0,1]^{A}$ be defined by $\phi(x)(f)=f(x)$ for all $x \in X$ and $f \in A$. Let $\pi_{f}:[0,1]^{A} \rightarrow[0,1]$ be the natural projection mapping for $f \in A$. Then $\pi_{f} \circ \phi=f$ is a continuous map on $X$ and so, $\phi$ is a continuous map. Let $Y=\phi(X)$. Then by Lemma 1.3.18, we have $w(Y) \leq|A| \leq \kappa$. To see that $\phi$ is one-to-one, let $x, y \in X$ with $x \neq y$. Then by Lemma 1.3.19, there exists $f \in A$ with $f(x) \neq f(y)$. Thus, $\phi(x)(f)=f(x) \neq f(y)=\phi(y)(f)$. Therefore, $i w(X) \leq n w(X)$.

(4) To see that $t(X) \leq \chi(X)$, suppose that $\chi(X) \leq \kappa$ for some infinite cardinal $\kappa$. Let $A \subseteq X$ such that $x \in \bar{A}$. Let $\mathcal{B}$ be a local base at $x$ such that $|\mathcal{B}| \leq \kappa$. For each $U \in \mathcal{B}$ pick $x_{U} \in U \cap A$. Then, let $B=\left\{x_{U}: U \in \mathcal{B}\right\}$. Clearly, $B \in A$ and $|B| \leq \kappa$. Now, let $x \in V \in \mathcal{T}$. Since $\mathcal{B}$ is a local base at $x$, there is $U \in \mathcal{B}$ such 
that $U \subseteq V$ and so, $x_{U} \in V \cap A$. Thus, $V \cap A \neq \emptyset$ for any $V \in \mathcal{T}$ with $x \in V$. Thus, $x \in \bar{B}$ and $t(X) \leq \chi(X)$.

To see that $\chi(X) \leq w(X)$, let $\mathcal{B}$ be a base of $X$. Then for any $x \in X$, the set $\mathcal{B}_{x}=\{U \in \mathcal{B}: x \in U\}$ forms a local base at $x$. Since $\mathcal{B}_{x} \subseteq \mathcal{B}$, we have that $\left|\mathcal{B}_{x}\right| \leq|\mathcal{B}|$, proving $\chi(X) \leq w(X)$.

Suppose $n w(X) \leq \kappa$ for some infinite cardinal $\kappa$. Let $A \subseteq X$ and $x \in \bar{A}$. Let $\mathcal{N}$ be a network of $X$ and define $\mathcal{N}^{\prime}=\{N \in \mathcal{N}: N \cap A \neq \emptyset\}$. Choose $a_{N} \in N \cap A$ for each $N \in \mathcal{N}^{\prime}$. The let $B=\left\{a_{N}: N \in \mathcal{N}^{\prime}\right\}$. Clearly, $B \subseteq A$. Also, $|B| \leq|\mathcal{N}| \leq \kappa$. To see that $x \in \bar{B}$, suppose that it is not. Then there is a $U \in \mathcal{T}$ with $x \in U$ such that $U \cap B \neq \emptyset$. Now, $B \cap A \neq \emptyset$ since $x \in \bar{A}$. Choose $a \in A \cap U$ and $N \in \mathcal{N}$ such that $a \in \mathcal{N} \subseteq U$. Then $N \in \mathcal{N}^{\prime}$. But this implies that $a \in B \cap U$, a contradiction. Thus, $x \in \bar{B}$ and $t(X) \leq n w(X)$.

\subsection{Compactness Properties}

The following section visits several types of compactness. The goal of this section is to familiarize the reader with these types of compactness as they will be used often in the remainder of the thesis. In addition to these, more specific types will be introduced throughout later chapters as necessary.

Definition 1.4.1. Let $X$ be a topological space and $A \subseteq X$. Then $A$ is compact if every open cover of $A$ has a finite subcover.

Definition 1.4.2. Let $X$ be a topological space and $A \subseteq X$. Then $A$ is countably compact if every countable open cover of $A$ has a finite subcover.

Definition 1.4.3. If $\mathcal{U}$ and $\mathcal{V}$ are covers of $X$, we say $\mathcal{U}$ refines $\mathcal{V}$, and write $\mathcal{U}<\mathcal{V}$, if and only if each $U \in \mathcal{U}$ is contained in some $V \in \mathcal{V}$. We say $\mathcal{U}$ is a refinement of $\mathcal{V}$.

Definition 1.4.4. A collection $\mathcal{U}$ of subsets of $X$ is locally finite if and only if each $x \in X$ has a neighborhood meeting only finitely many $U \in \mathcal{U}$.

Definition 1.4.5. A collection $\mathcal{U}$ of subsets of $X$ is point finite if and only if each $x \in X$ belongs to only finitely many $U \in \mathcal{U}$.

Definition 1.4.6. A Hausdorff space $X$ is paracompact if and only if each open cover of $X$ has an open locally finite refinement. 
Definition 1.4.7. A space is metacompact if and only if each open cover has an open point finite refinement.

Definition 1.4.8. A space is called $\sigma$-compact ( $\sigma$-countably compact) if it is the union of a countable set of compact (countably compact) subspaces.

Definition 1.4.9. A space is called pseudocompact if every real-valued continuous function on it is bounded. 


\section{Chapter 2}

\section{Introduction to $C_{p}(X)$}

The goal of this chapter is to introduce the reader to the basic ideas and concepts relating to $C_{p}$-theory. As mentioned in Chapter 1, all spaces will be taken to be Tychonoff. The reason being that Tychonoff is needed to separate points.

\subsection{Definitions}

The following section contains several terms that provide the reader with an introduction to function spaces and various topologies that can be placed on these spaces [1].

Definition 2.1.1. The set of all continuous maps from a space $X$ into a space $Y$ is denoted by $C(X, Y)$.

Note that we will write $C(X, \mathbb{R})$ as $C(X)$.

Definition 2.1.2. The set of all functions, $f \in C(X)$, such that $f$ is bounded, is denoted by $C^{0}(X)$.

Definition 2.1.3. $C_{p}(X)$ and $C_{p}^{0}(X)$ are used to represent $C(X)$ and $C^{0}(X)$ endowed with the topology of pointwise convergence.

Definition 2.1.4. Let $\mathcal{E}$ be the family of all finite subsets of $X$. Then $\mathcal{T}_{\mathcal{E}}$ is the topology of pointwise convergence. $\left(C(X, Y), \mathcal{T}_{\mathcal{E}}\right)=C_{p}(X, Y)$.

Definition 2.1.5. Let $\mathcal{E}$ be the family of all compact subsets of $X$. Then $\mathcal{T}_{\mathcal{E}}$ is the compact-open topology. $\left(C(X, Y), \mathcal{T}_{\mathcal{E}}\right)=C_{c}(X, Y)$.

Definition 2.1.6. Let $\mathcal{E}$ be the family of all bounded subsets in $X$. Then

$$
\left(C(X, Y), \mathcal{T}_{\mathcal{E}}\right)=C_{0}(X, Y) .
$$




\section{$2.2 \quad$ Properties}

In this section we will examine some basic properties of $C_{p}(X)$. These properties will familiarize us with $C_{p}(X)$ spaces and provide a better understanding of the way in which these spaces work. The proofs in this section are modified versions of those found in [9] and [1].

Proposition 2.2.1. Let $X$ and $Z$ be topological spaces and $Y \subseteq Z$, then the topology of $C_{p}(X, Y)$ coincides with the topology on $C(X, Z)$ induced from $C_{p}(X, Y)$ and so $C_{p}(X, Y) \subseteq C_{p}(X, Z)$.

\section{Proof. Let}

$$
A=\left\{x_{1}, x_{2}, \ldots, x_{n} ; U_{1}, U_{2}, \ldots, U_{n}\right\}=\left\{f \in C_{p}(X, Z): f\left(x_{i}\right) \in U_{i} \text { for all } i \leq n\right\}
$$

be a basic open set in $C_{p}(X, Z)$. Then

$$
A \cap C_{p}(X, Y)=\left\{f \in C_{p}(X, Y): f\left(x_{i}\right) \in U_{i} \cap Y \text { for all } i \leq n\right\}
$$

and $A \cap C_{p}(X, Y)$ is an open set in $C_{p}(X, Y)$.

Let

$$
W=\left\{x_{1}, \ldots, x_{n} ; U_{1}, \ldots, U_{n}\right\}=\left\{f \in C_{p}(X, Y): f\left(x_{i}\right) \in U_{i} \text { for all } i \leq n\right\}
$$

be a basic open set of $C_{p}(X, Y)$. For each $i \leq n$, let $O_{i} \in \mathcal{T}_{Z}$ such that $U_{i}=O_{i} \cap Y$. Let

$$
W^{\prime}=\left\{x_{1}, \ldots, x_{n} ; O_{1}, \ldots, O_{n}\right\}=\left\{f \in C_{p}(X, Z): f\left(x_{i}\right) \in O_{i} \text { for all } i \leq n\right\} .
$$

Then $W^{\prime}=W \cap C_{p}(X, Y)$ and $W^{\prime}$ is a basic open set in $C_{p}(X, Z)$.

Proposition 2.2.2. If $X, Z$ are topological spaces and $Y \subseteq Z$ is closed, then $C_{p}(X, Y)$ is a closed subspace of $C_{p}(X, Z)$.

Proof. Let $f \in C_{p}(X, Z) \backslash C_{p}(X, Y)$. Then there exists an $x \in X$ such that $f(x) \in$ $Z \backslash Y$. Let $U_{f}=\left\{f \in C_{p}(X, Z): f(x) \in Z \backslash Y\right\}$. Then $U_{f}$ is open in $C_{p}(X, Z)$. Furthermore, $f \in U_{f} \subseteq C_{p}(X, Z) \backslash C_{p}(X, Y)$. Therefore $C_{p}(X, Z) \backslash C_{p}(X, Y)=\bigcup\left\{U_{f}\right.$ : $\left.f \in C_{p}(X, Z) \backslash C_{p}(X, Y)\right\}$ which is open. Thus, $C_{p}(X, Y)$ is a closed subset.

The topological product $\mathbb{R}^{X}$ is the set of all maps from $X$ into $\mathbb{R}$ endowed with the topology of pointwise convergence. By definition of $C_{p}(X)$, we see that $C_{p}(X) \subseteq \mathbb{R}^{X}$. Furthermore, $C_{p}(X)$ is everywhere dense in $\mathbb{R}^{X}$.

The following theorems and propositions illustrate how properties of a space $X$ are characterized by topological properties of $C_{p}(X)$. 
Theorem 2.2.3. For any infinite space $X$,

$$
|X|=\chi\left(C_{p}(X)\right)=w\left(C_{p}(X)\right) .
$$

Proof. Let $\mathcal{B}=\left\{\left[x_{1}, \ldots, x_{k} ; U_{1}, \ldots, U_{k}\right]: k \in \mathbb{N}, x_{i} \in X\right.$, and $U_{i}$ is a rational interval for $i \leq k\}$ be a base for $C_{p}(X)$. Then $|\mathcal{B}| \leq|X|$, since $\mathcal{B}$ is a base for $C_{p}(X)$ and the topology on $C_{p}(X)$ is created from the finite subsets of $X$. Thus, we have that $w\left(C_{p}(X)\right) \leq|X|$.

Now, let $h: X \rightarrow \mathbb{R}$ be defined by $h(x)=0$ for all $x \in X$, and let $\mathcal{C}$ be a local base at $h$ in $C_{p}(X)$. For any $V \in \mathcal{C}$, there is a basic open set $U(V)$ with $h \in U(V) \subseteq V$. Then $\{U(V): V \in \mathcal{C}\}$ also forms a local at $h$. Thus, it may be assumed without loss of generality that the elements of $\mathcal{C}$ are basic. Given $V=\left[x_{1}, \ldots, x_{k} ; U_{1}, \ldots, U_{k}\right] \in \mathcal{C}$, let $\operatorname{supp}(V)=\left\{x_{1}, \ldots, x_{k}\right\}$ and let $Y=\cup\{\operatorname{supp}(V): V \in \mathcal{C}\}$, where $\operatorname{supp}(V)$ is the support. If $x \in X \backslash Y$, then there is $V=\left[x_{1}, \ldots, x_{k} ; U_{1}, \ldots, U_{k}\right] \in \mathcal{C}$ with $V \subseteq W=[x ;(-1,1)]$. However, $\operatorname{supp}(V)=\left\{x_{1}, \ldots, x_{k}\right\} \subseteq Y \subseteq X \backslash\{x\}$. So there exists a function $f \in C_{p}(X)$ such that $f\left(x_{i}\right) \in U_{i}$ and $f(x)=1$. Then $f \in V \backslash W$, which gives a contradiction. Thus, $X=Y$ and so $|X|=|Y| \leq \chi\left(C_{p}(X)\right)$. Also, by Theorem 1.3.20, we have that $w\left(C_{p}(X)\right) \leq|X| \leq \chi\left(C_{p}(X)\right) \leq w\left(C_{p}(X)\right)$. Therefore, $|X|=\chi\left(C_{p}(X)\right)=w\left(C_{p}(X)\right)$.

Theorem 2.2.4. For a space $X, n w(X)=n w\left(C_{p}(X)\right)$.

Proof. Let $\mathcal{N}$ be a network of the topological space $X$ such that $|\mathcal{N}|=n w(X)$. For any collection $N_{1}, \ldots, N_{k} \in \mathcal{N}$ and any open intervals with rational end points $I_{1}, \ldots, I_{k}$, let $M\left(N_{1}, \ldots, N_{k} ; I_{1}, \ldots, I_{k}\right)=\left\{f \in C_{p}(X): f\left(N_{j}\right) \subseteq I_{j}\right.$ for all $\left.j \leq k\right\}$. Now, let $\mathcal{M}=\left\{M\left(N_{1}, \ldots, N_{k} ; I_{1}, \ldots, I_{k}\right): n \in \mathbb{N}, N_{1}, \ldots, N_{k} \in \mathcal{N}\right.$, and $I_{j}$ is a rational interval for all $j \leq k\}$. Clearly, $|\mathcal{M}| \leq n w(X)$. To see that $\mathcal{M}$ is a network of $C_{p}(X)$, let $f \in C_{p}(X)$ and $f \in U \in \mathcal{T}_{\left(C_{p}(X)\right)}$. There is a canonical open set $V=\left[x_{1}, \ldots, x_{k} ; O_{1}, \ldots, O_{k}\right]$ with $f \in V \subseteq U$. Without loss of generality, consider $x_{1}, \ldots, x_{k}$ distinct. Since $f$ is continuous, we can choose disjoint open sets $U_{1}, \ldots, U_{k}$ such that $x_{i} \in U_{i}$ for each $i \leq k$ and rational intervals $I_{1}, \ldots, I_{k}$ such that $f\left(U_{j}\right) \subseteq$ $I_{j} \subseteq O_{j}$ for all $j \leq k$. Then there exist $N_{1}, \ldots, N_{k} \in \mathcal{N}$ such that $x_{j} \in N_{j} \subseteq U_{j}$ for all $j \leq k$. Thus, $f \in M\left(N_{1}, \ldots, N_{k} ; I_{1}, \ldots, I_{k}\right) \subseteq V \subseteq U$, and $\mathcal{M}$ is a network of $C_{p}(X)$. Since $|\mathcal{M}| \leq n w(X)$, we have $n w\left(C_{p}(X)\right) \leq n w(X)$ for any space $X$. Thus, $n w\left(C_{p}\left(C_{p}(X)\right)\right) \leq n w\left(C_{p}(X)\right) \leq n w(X)$ for any space $X$. But, $X$ can be embedded in $C_{p}\left(C_{p}(X)\right)$ which tells us $n w(X) \leq n w\left(C_{p}\left(C_{p}(X)\right)\right)$. Combining these inequalities, we have that $n w(X)=n w\left(C_{p}(X)\right)$.

Theorem 2.2.5. $d(X)=\psi\left(C_{p}(X)\right)=\Delta\left(C_{p}(X)\right)=i w\left(C_{p}(X)\right)$. 
Proof. Let $Y$ be a topological space and $\gamma \subseteq \mathcal{T}_{Y \times Y}$ be a family such that $\Delta_{Y}=\cap \gamma$. Fix $y \in Y$ and for $U \in \gamma$, choose $O_{U} \in \mathcal{T}_{Y}$ with $y \in O_{U}$ such that $O_{U} \times O_{U} \subseteq U$. Then $\cap\left\{O_{U}: U \in \gamma\right\}=\{y\}$. Therefore, $\psi(Y) \leq \Delta(Y)$.

Suppose that $f: Z \rightarrow Y$ is a condensation and $\mathcal{B}$ is a base for $Y$ such that $|\mathcal{B}|=$ $\kappa=i w(X)$. Then $l\left((Y \times Y) \backslash \Delta_{Y}\right) \leq w\left((Y \times Y) \backslash \Delta_{Y}\right) \leq \kappa$. For each $z \in(Y \times Y) \backslash \Delta_{Y}$, choose $U_{z} \in \mathcal{T}_{Y \times Y}$ with $z \in U_{z}$ such that $\overline{U_{z}} \cap \Delta_{Y}=\emptyset$. Let $\mathcal{U}=\left\{U_{z}: z \in(Y \times Y) \backslash \Delta_{Y}\right\}$ be an open cover of the $(Y \times Y) \backslash \Delta_{Y}$ with a subcover $\mathcal{V}$ such that $|\mathcal{V}| \leq \kappa$. Then, $(Y \times Y) \backslash \Delta_{Y}=\cup\{\bar{U}: U \in \mathcal{V}\}$ is a union of less than or equal to $\kappa$ closed set. Thus, $\psi\left(\Delta_{Y}, Y \times Y\right) \leq \kappa$. Since $f$ is a condensation, $\psi\left(\Delta_{Z}, Z \times Z\right) \leq \kappa$ and so, $\Delta(Z) \leq i w(Z)$.

Given a basic set $V=\left[x_{1}, \ldots, x_{n} ; O_{1}, \ldots, O_{n}\right] \in \mathcal{T}_{C_{p}(X)}$, let $\operatorname{supp}(V)=\left\{x_{1}, \ldots, x_{n}\right\}$. Let $\gamma$ be any subset of $\mathcal{T}_{C_{p}(X)}$ such that $\{u\}=\cap \gamma$, where $u \equiv 0$ and $|\gamma|=\kappa$. For each $U \in \gamma$ let $O_{U}=\left[x_{1}, \ldots, x_{n} ; O_{1}, \ldots, O_{n}\right]$ be a basic open set such that $u \in O_{U} \subseteq U$. Then $Y=\cup\left\{\operatorname{supp}\left(O_{U}\right): U \in \gamma\right\}$ and $|Y| \leq \kappa$. If $X \neq \bar{Y}$, choose $x \in X \backslash \bar{Y}$ and $f \in C_{p}(X)$ such that $f(x)=1$ and $f(Y) \subseteq\{0\}$. Then $f \in \cap \gamma$, a contradiction to $f \neq u$ and $\cap \gamma=\{u\}$. Thus $Y$ is dense in $X$ and $d(X) \leq|Y| \leq \kappa$. Therefore, $d(X) \leq \psi\left(C_{p}(X)\right)$, and so we have $d(X) \leq \psi\left(C_{p}(X)\right) \leq \Delta\left(C_{p}(X)\right) \leq i w\left(C_{p}(X)\right)$.

To see that $i w\left(C_{p}(X)\right) \leq d(X)$, fix $Y \subseteq X$ such that $|Y|=d(X)$ and $X=\bar{Y}$. Let $\pi_{Y}: C_{p}(X) \rightarrow Z=\pi_{Y}\left(C_{p}(X)\right) \subseteq C_{p}(Y)$ be the restriction map $\left(\pi_{Y}(f)=\left.f\right|_{Y}\right)$. Then $\pi_{Y}$ is a condensation and $w(Z) \leq w\left(C_{p}(Y)\right)=|Y|=d(X)$. Therefore, $i w\left(C_{p}(X)\right) \leq$ $d(X)$.

Using the definitions of our cardinal invariants, and the result proved above, we can summarize the previous theorem as $C_{p}(X)$ condenses onto a second countable space if and only if $X$ is separable.

Theorem 2.2.6. $i w(X)=d\left(C_{p}(X)\right)$

Proof. Note that $X$ embeds $C_{p}\left(C_{p}(X)\right)$. So, we have that $i w(X) \leq i w\left(C_{p}\left(C_{p}(X)\right)\right) \leq$ $d\left(C_{p}(X)\right)$ by that and the result proved in the previous theorem.

To see that $d\left(C_{p}(X)\right) \leq i w(X)$, suppose $i w(X) \leq \kappa$. Then take a condensation $f: X \rightarrow Y$ such that $w(Y) \leq \kappa$. Then the dual mapping $f^{*}$ embeds $C_{p}(Y)$ into $C_{p}(X)$ as a dense subspace. Let $Z=f^{*}\left(C_{p}(Y)\right)$. Then $d(Z) \leq n w(Z)=n w\left(C_{p}(Y)\right)=$ $n w(Y) \leq w(Y) \leq \kappa$. Therefore, $d\left(C_{p}(X)\right) \leq d(Z) \leq \kappa$, and so $d\left(C_{p}(X)\right) \leq i w(X)$.

Using the definitions and the result of the preceding theorem, we can summarize that $C_{p}(X)$ is separable if and only if $X$ condenses onto a second countable space. 


\section{$2.3 \quad$ Examples}

In this section, we will look at three familiar spaces - the real line, the Sorgenfrey line and the Moore-Niemytzki Plane - and some of the properties of their corresponding $C_{p}$-spaces. The proofs in this section are modified versions of the ones in [9].

Theorem 2.3.1. The set of all increasing functions is closed in $C_{p}(\mathbb{R})$.

Proof. Let $\mathcal{I} \subseteq C_{p}(\mathbb{R})$ be the set of all of increasing functions. For $f \in C_{p}(\mathbb{R}) \backslash \mathcal{I}$ there are $x, y \in X$ such that $x<y$ and $f(x)>f(y)$. Let $d=f(x)-f(y)$. Let $U=\left[x, y ;\left(f(x)-\frac{d}{3}, f(x)+\frac{d}{3}\right),\left(f(y)-\frac{d}{3}, f(y)+\frac{d}{3}\right)\right]$. Then $U$ is open in $C_{p}(\mathbb{R})$ and $f \in U$. Let $g \in U$. Then $g(y)<f(y)+\frac{d}{3}<f(x)-\frac{d}{3}<g(x)$. Thus $g(y)<g(x)$ and so $g$ is not increasing. Also, $U \cap \mathcal{I}=\emptyset$. Since each $f \in C_{p}(\mathbb{R}) \backslash \mathcal{I}$ has a neighborhood that is also contained in $C_{p}(\mathbb{R}) \backslash \mathcal{I}, C_{p}(\mathbb{R}) \backslash \mathcal{I}$ is open and $\mathcal{I}$ is closed.

Using a nearly identical proof as the theorem above, we get the following result.

Corollary 2.3.2. The set of all decreasing functions is closed in $C_{p}(\mathbb{R})$.

The following theorem will provide a further look into $C_{p}(\mathbb{R})$, comparing density and network weight.

Theorem 2.3.3. There exists a countable $A \subseteq C_{p}(\mathbb{R})$ such that $\bar{A}=C_{p}(\mathbb{R})$.

Proof. Let $\mathcal{B}=\left\{\left\{O_{1}, \ldots, O_{n}\right\}: n \in \mathbb{N}, O_{i}=\left(a_{i}, b_{i}\right)\right.$ is a rational open interval for all $i \leq n$ and $\left[a_{i}, b_{i}\right] \cap\left[a_{j}, b_{j}\right]=\emptyset$ if $\left.i \neq j\right\}$. Given that $O=\left\{O_{1}, \ldots, O_{n}\right\} \in \mathcal{B}$, let $m(O)=n$. If $q=\left(q_{1}, \ldots, q_{n}\right)$ is an $n$-tuple of rational numbers, fix $f_{O, q} \in C_{p}(\mathbb{R})$ such that $f_{O, q}\left(O_{i}\right)=\left\{q_{i}\right\}$ for all $i \leq n$. Then the set $A=\left\{f_{O, q}: O \in \mathcal{B}\right.$ and $q$ is an $m(O)-$ tuple of rationals $\}$ is countable. To see that $\bar{A}=C_{p}(\mathbb{R})$, given a basic open set $W=\left[x_{1}, \ldots, x_{n} ; U_{1}, \ldots, U_{n}\right]$ choose $q_{i} \in U_{i} \cap \mathbb{Q}$ for all $i \leq n$ and let $q=\left(q_{1}, \ldots, q_{n}\right)$. Then, there exists $O=\left\{O_{1}, \ldots, O_{n}\right\} \in \mathcal{B}$ such that $x_{i} \in O_{i}$ for all $i \leq n$. Then $f_{O, q} \in W \cap A$. Thus, $\bar{A}=C_{p}(\mathbb{R})$.

Another way of looking at the preceding theorem is that

$$
d\left(C_{p}(\mathbb{R})\right) \leq n w\left(C_{p}(\mathbb{R})\right) \leq n w(\mathbb{R})=\aleph_{0}
$$

The following two corollaries focus on subsets of the reals, namely, the rationals and natural numbers. 
Corollary 2.3.4. $C_{p}(\mathbb{Q})$ has a countable base.

Proof. Let $\mathcal{U}$ be the family of all open intervals in $\mathbb{R}$ that have rational endpoints. Consider the family $\mathcal{B}$ of all sets $\left[x_{1}, \ldots, x_{n} ; O_{1}, \ldots, O_{n}\right]$ where $n \in \mathbb{N}, x_{i} \in \mathbb{Q}$ and $O_{i} \in \mathcal{U}$ for all $i \leq n$. Then $\mathcal{B}$ forms a countable base for $C_{p}(\mathbb{Q})$.

Corollary 2.3.5. $C_{p}(\mathbb{N})$ has a countable base.

Proof. Let $\mathcal{U}$ be the family of all open intervals in $\mathbb{R}$ that have rational endpoints. Consider the family $\mathcal{B}$ of all sets $\left[x_{1}, \ldots, x_{n} ; O_{1}, \ldots, O_{n}\right]$ where $n \in \mathbb{N}, x_{i} \in \mathbb{N}$ and $O_{i} \in \mathcal{U}$ for all $i \leq n$. Then $\mathcal{B}$ forms a countable base for $C_{p}(\mathbb{N})$.

The following examples deal with two common spaces: the Sorgenfrey line and the Moore-Niemytzki Plane.

Definition 2.3.6. The real line with the topology generated by $\{[a, b): a, b \in \mathbb{R}, a<$ $b\}$ is called the Sorgenfrey line. We will denote the Sorgenfrey line by the notation $(S, \mathcal{T})$.

Proposition 2.3.7. Let $X$ be a separable space with $\operatorname{ext}(X) \geq \mathfrak{c}$. Then $X$ is not normal.

Proof. Let $S \subseteq X$ be closed discrete with $|S|=\mathfrak{c}$. Since $X$ is separable, we know there is a countable dense $D \subseteq X$. Then $|\mathcal{P}(D)|=\mathfrak{c}<|\mathcal{P}(S)|$. Thus, by Jones' Lemma, $X$ is not normal.

Proposition 2.3.8. The space $S \times S$ has a closed discrete subspace of cardinality continuum.

Proof. Let $D=\{(t,-t): t \in \mathbb{R}\} \subseteq S \times S$. Now for any $d=(t,-t) \in D$, the set $U=[t, t+1) \times[-t,-t+1)$ is open in $S \times S$. Also, $U \cap D=\{d\}$, and so $D$ is discrete. Now if $z=(x, y) \notin D$, then there is $\varepsilon=|x+y|>0$. Thus, $W=\left[x, x+\frac{\varepsilon}{2}\right) \times\left[y, y+\frac{\varepsilon}{2}\right)$ is an open neighborhood of $S \times S$ with $W \cap D=\emptyset$. Thus, $D$ is closed in $S \times S$ and $\operatorname{ext}(S \times S)=\mathfrak{c}$. 
Proposition 2.3.9. Let $X, Y$ be topological spaces. Given a continuous map $r$ : $X \rightarrow Y$, define the dual map $r^{*}: C_{p}(X) \rightarrow C_{p}(Y)$ by $r^{*}(f)=f \circ r$ for any $f \in C_{p}(Y)$. If $r(X)=Y$, then $r^{*}\left(C_{p}(Y)\right)$ is dense in $C_{p}(X)$ if and only if $r$ is a condensation.

Proof. Suppose $r: X \rightarrow Y$ is a condensation. Let $f \in C_{p}(X), x_{1}, \ldots, x_{n} \in X$ and $\varepsilon>0$ be given. Then there exists $g \in C_{p}(Y)$ such that $g\left(r\left(x_{i}\right)\right)=f\left(x_{i}\right)$ for all $i \leq n$. Then $r^{*}(g)=g \circ r$ is an element of the basic open neighborhood $O_{X}\left(f, x_{1}, \ldots, x_{n}, \varepsilon\right)=$ $\left\{h \in C_{p}(X):\left|h\left(x_{i}\right)-f\left(x_{i}\right)\right|<\varepsilon\right.$ for all $\left.i \leq n\right\}$. Thus, $f \in \overline{r^{*}\left(C_{p}(Y)\right)}$ for each $f \in C_{p}(X)$. Thus, $r^{*}\left(C_{p}(Y)\right)=C_{p}(X)$.

To see that if $r^{*}\left(C_{p}(Y)\right)$ is dense in $C_{p}(X)$ then $r$ is a condensation, suppose $r$ is not one-to-one. Let $x, y \in X$ with $x \neq y$ such that $r(x)=r(y)$. Then $U_{X}=$ $[x, y ;(0,1),(2,3)]$ is a non-empty open set in $C_{p}(X)$. However, $U_{X} \cap r^{*}\left(C_{p}(Y)\right)=\emptyset$. Thus $r^{*}\left(C_{p}(Y)\right)$ is not dense in $C_{p}(X)$.

Theorem 2.3.10. $C_{p}(S)$ is not normal.

Proof. Let $r: \mathbb{R} \rightarrow \mathbb{R}$ be the identity mapping for all $t \in \mathbb{R}$. Then $r: S \rightarrow \mathbb{R}$ is a condensation. Thus, $r\left(C_{p}(\mathbb{R})\right)$ is a dense subspace of $C_{p}(S)$ which is homeomorphic to $C_{p}(\mathbb{R})$. If $A$ is a countable dense subspace of $C_{p}(\mathbb{R})$, then $r^{*}(A)$ is a countable dense subspace of $C_{p}(S)$. Hence $C_{p}(S)$ is separable.

Let $[a, b) \subseteq S$. Let $f(t)=t-a$ and $g(t)=\frac{1}{b-a} \cdot t$ for all $t \in \mathbb{R}$. Then $h=g \circ f$ : $S \rightarrow S$ is a homeomorphism. Also, $h([a, b))=[0,1)$. Thus, any two clopen intervals in $S$ are homeomorphic subspaces. Now $S=\cup\left\{A_{k}: k \in \omega\right\}$ where $A_{2 i}=[-i-1,-i)$ and $A_{2 i+1}=[i, i+1)$ for all $i \in \omega$. Thus, since $S$ can be represented as a union of a family $\left\{A_{k}: k \in \omega\right\}$ of pairwise disjoint open subsets of $S$, then $S$ is homeomorphic to $\bigoplus\left\{A_{k}: k \in \omega\right\}$. Notice that similarly, we can write $[0,1)=\cup\left\{\left[p_{i}, q_{i}\right): i \in \omega\right\}$ where $p_{i}=\frac{i}{i+1}$ and $q_{i}=\frac{i+1}{i+2}$ for all $i \in \omega$. Thus, $[0,1)=\bigoplus\left\{B_{k}: k \in \omega\right\}$ where $B_{k}=\left[p_{k}, q_{k}\right)$. This implies that $S$ is homeomorphic to any of its clopen intervals. Let us take $S^{\prime}=[0,1)$ for the remainder of the proof.

Now, for each $t \in(0,1]$, let $f_{t}(x)=1$ if $0 \leq x<t$ and $f_{t}(x)=0$ for all $x \in[t, 1)$. Then $f_{t} \in C_{p}\left(S^{\prime}\right)$ for all $t \in[0,1)$.

Claim 1. The set $F=\left\{f_{t}: t \in(0,1]\right\}$ is closed in $C_{p}\left(S^{\prime}\right)$.

Proof. Given that $f \notin F$, suppose $f(x) \notin\{0,1\}$ for some $x \in S^{\prime}$. Then for $\varepsilon=\min \{|f(x)|,|f(x)-1|\}$ the set $[x ;(f(x)-\varepsilon, f(x)+\varepsilon)]$ is an open neighborhood of $f$ that does not meet $F$. If $f\left(S^{\prime}\right) \subseteq\{0,1\}$ and $f \in \bar{F}$, then if $f(x)=1$ then $f(y)=1$ for $y<x$. For if $f(y)=0$ for some $y<x$, then $\left[y, x:\left(-\frac{1}{2}, \frac{1}{2}\right),\left(\frac{1}{2}, \frac{3}{2}\right)\right]$ is an open neighborhood of $f$ that does not meet $F$ since all functions from $F$ are non-increasing. By similar reasoning, $f(x)=0$ tells us $f(y)=0$ for $y>x$. Then, there exists $t \in S^{\prime}$ such that $f(x)=1$ for all $x<t$ and $f(x)=0$ for all $x>t$. If $f(t)=1$, then $f$ is discontinuous, so $f(t)=0$ and $f=f_{t} \in F$. Therefore, $F$ is closed in $C_{p}\left(S^{\prime}\right)$. 
Claim 2. The map $\phi: S^{\prime} \rightarrow F$ defined by $\phi(s)=f_{1-s}$ is a homeomorphism.

Proof. Clearly, $\phi$ is one-to-one and onto. To prove the continuity of $\phi$, it suffices to show that $h_{t}=\pi_{t} \circ \phi$ is continuous for each $\pi_{t}$ defined by $\pi_{t}(f)=f(t)$ for all $f \in C_{p}\left(S^{\prime}\right)$ and $t \in[0,1)$, since $C_{p}\left(S^{\prime}\right)$ is a subspace of $\mathbb{R}^{S^{\prime}}$. Since $h_{t}(s)=f_{1-s}(t)=1$ for $s<1-t$ and $h_{t}(s)=f_{1-s}(t)=0$ for $s \geq 1-t, h_{t}$ is continuous for all $t \in S^{\prime}$. Finally, to see that $\phi$ is a homeomorphism, it need only be shown that $\phi$ is open. Since the clopen intervals form a base of $S^{\prime}$, it suffices to show that $\phi(U)$ is open in $F$ for any $U=[a, b) \subseteq S^{\prime}$. Now, $\phi(U)=\left\{f_{t}: 1-b<t \leq 1-a\right\}=\left[1-b, 1-a ;\left(\frac{1}{2}, \frac{3}{2}\right),\left(-\frac{1}{2}, \frac{1}{2}\right)\right] \cap F$ which is open in $F$. Thus, the mapping $\phi$ is a homeomorphism.

Claim 3. The spaces $C_{p}\left(S^{\prime}\right)$ and $C_{p}\left(S^{\prime}\right) \times C_{p}\left(S^{\prime}\right)$ are homeomorphic.

Proof. Now $S^{\prime}=\left[0, \frac{1}{2}\right) \cup\left[\frac{1}{2}, 1\right)$ and since $S^{\prime}$ is homeomorphic to every arrow, we know that the space $S^{\prime}$ is homeomorphic to $S^{\prime} \oplus S^{\prime}$. Then $C_{p}\left(S^{\prime}\right)$ is homeomorphic to $C_{p}\left(S^{\prime} \oplus S^{\prime}\right)$. But, $C_{p}\left(S^{\prime} \oplus S^{\prime}\right)$ is homeomorphic to $C_{p}\left(S^{\prime} \times S^{\prime}\right)$ and so $C_{p}\left(S^{\prime}\right)$ is homeomorphic to $C_{p}\left(S^{\prime} \times S^{\prime}\right)$.

From Claims 1 and 2, we see that $F$ is a closed subspace of $C_{p}\left(S^{\prime}\right)$ homeomorphic to $S^{\prime}$. And so, $C_{p}(S)$ has some closed subspace $T$ that is homeomorphic to $S$. Then, the space $T \times T$ is closed subspace of $C_{p}(S) \times C_{p}(S)$. Now, we know there is a closed discrete $D \subseteq T \times T$ with $|D|=\mathfrak{c}$. Then $D$ is a closed discrete subspace of $C_{p}(S) \times C_{p}(S)$ as well. By Claim 3, we know that $C_{p}(S)$ is homeomorphic to $C_{p}(S) \times C_{p}(S)$. Thus, $C_{p}(S)$ has a closed discrete subspace with cardinality of the continuum and so is not normal.

Definition 2.3.11. Let $\Gamma=\mathbb{R} \times[0, \infty)$ and $S=\mathbb{R} \times\{0\}$. Also, let $d$ denote the usual metric on $\mathbb{R} \times \mathbb{R}$. For each $x \in \Gamma \backslash S$, let $\mathcal{B}_{x}=\left\{B_{d}(x, r): r<d(x, S)\right\}$. Now suppose that $x=(s, 0) \in S$. Then $\mathcal{B}_{x}=\left\{B_{d}((s, r), r) \cup\{x\}: r>0\right\}$. Let $\mathcal{B}=\cup_{x \in \Gamma} \mathcal{B}_{x}$. Let $\mathcal{T}$ be the topology on $\Gamma$ generated by $\mathcal{B}$. Then $(\Gamma, \mathcal{T})$ is called the Moore-Niemytzki Plane.

The proof of the following theorem can be found in full in [9].

Theorem 2.3.12. $C_{p}(\Gamma)$ is not normal. 


\section{Chapter 3}

\section{$C_{p}(X)$ and Lindelöfness}

In this chapter, we will explore two open questions in $C_{p}$-theory originally posed by Arhangel'skii in [1]. The results provided in this chapter are the work of many mathematicians. The first question:

Question. Does there exist a natural topological property of $C_{p}(X)$ which would characterize whether the space $X$ is Lindelöf?

The second question is again a Lindelöf question, however, this one pertains to the product topology:

Question. Suppose $C_{p}(X)$ is Lindelöf. Is it true that the space $C_{p}(X) \times C_{p}(X)$ is Lindelöf?

Unfortunately, the answers to these questions for general spaces do not exist. In the following chapter, we will take a look at specific spaces in regards to the above mentioned questions.

For the theorems we will be discussing in the remaining chapters, we will often need to leave ZFC and explore other models of set theory. We begin by mentioning a consequence of the Continuum Hypothesis, namely, Luzin's Axiom which states that $2^{\omega_{1}}>\mathfrak{c}$.

The following theorem and corollary were proven by Tkachenko and Tkachuk and can be found in [11] with complete proofs in [8].

Theorem 3.0.13. If $X$ is Lindelöf and $w(X)<\mathfrak{c}$, then under Luzin's Axiom every compact continuous image of $C_{p}(X)$ is metrizable.

Corollary 3.0.14. Under Luzin's Axiom, if $X$ is a Lindelöf first countable space, then every compact continuous image of $C_{p}(X)$ is metrizable. 
In ZFC, the above theorem and corollary is not obtained, even if the conditions are changed to include a compact first countable space.

Next we look at a theorem which can be found in [9].

Theorem 3.0.15. $t\left(C_{p}(X)\right)=\sup \left\{l\left(X^{n}\right): n \in \mathbb{N}\right\}$. In particular, the tightness of $C_{p}(X)$ is countable if and only if $X^{n}$ is a Lindelöf space for any $n \in \mathbb{N}$.

This theorem is the motivation behind a question first asked by Arhangel'skii in $[2]$.

Question. Let $X$ be a Lindelöf space. Is it true that $C_{p}(X)$ condenses onto a space of countable tightness?

Going back to the second question presented in the chapter, we have the following two theorems proved by Okunev [6] and Reznichenko [1], respectively. Notice that both theorems are not ZFC, and instead MA $+\neg \mathrm{CH}$ which is taken to be Martin's Axiom with the negation of the Continuum Hypothesis.

Definition 3.0.16. A space $X$ is said to be $\omega$-monolithic if $n w(\bar{A}) \leq \omega$ for every $A \subseteq X$ such that $|A| \leq \omega$.

Theorem 3.0.17. (MA $+\neg \mathrm{CH}$ ) If $X^{n}$ is Lindelöf, for each $n \in \omega$, then every compact subspace of $C_{p}(X)$ is $\omega$-monolithic.

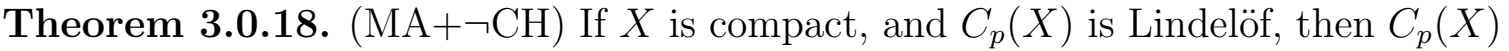
is $\omega$-monolithic.

This section will end with a conjecture made by Arhangel'skii in [2].

Conjecture. If $X$ is compact, and $C_{p}(X)$ is Lindelöf, then $C_{p}(X) \times C_{p}(X)$ is also Lindelöf. 


\section{Chapter 4}

\section{Lindelöfness of $C_{p}(X)$ Examples}

In the previous chapter, a general look was taken at two specific open questions originally asked by Arhangel'skii. In this chapter, we will consider more specific questions by putting constraints on the space $X$. The three spaces we will look at occur when $X$ has a single non-isolated point, when $X$ is a Michael space, and finally, when $X$ is a Mrówka space [5].

\section{1 $X$ has a Single Non-Isolated Point}

In this section, we take a look at the case when $X$ is a space with all but one of its points isolated. We begin by trying to find properties of this space $X$ that will make $C_{p}(X)$ Lindelöf. We begin with the following theorem and corollary proven by Malykhin and Leiderman in [4].

Theorem 4.1.1. Let $X$ be a space with a single non-isolated point. The following properties are equivalent:

1. $X$ is Lindelöf and for any finite $n$ the tightness of $X^{n}$ is countable.

2. $C_{p}(X)$ is Lindelöf.

3. $C_{p}(X, M)$ is Lindelöf for any separable metrizable space $M$.

A corollary to the preceding theorem considers the countable product of $C_{p}(X)$.

Corollary 4.1.2. Let $X$ be a topological space with a single non-isolated point. If $C_{p}(X)$ is Lindelöf, then the countable product $C_{p}(X)^{\omega}$ is also Lindelöf.

Finally, we consider the possibility of $C_{p}(X) \times C_{p}(Y)$ where $X$ and $Y$ are different spaces. To do so, we use different models of set theory besides ZFC.

The following theorem assuming the model of ZFC obtained by adding one Cohen real can also be found in [4]. 
Theorem 4.1.3. There are two spaces with a single non-isolated point $X$ and $Y$ such that both $C_{p}(X)$ and $C_{p}(Y)$ are Lindelöf but the product $C_{p}(X) \times C_{p}(Y)$ is not Lindelöf.

Our final theorems for this space involve the PFA or proper forcing axiom model of set theory. Both theorems are attributed to Todorcevic and can be found in [5].

Theorem 4.1.4. (PFA) Let $X$ and $Y$ be two spaces with at most one non-isolated point. If the spaces $C_{p}(X)$ and $C_{p}(Y)$ are Lindelöf, then the product $C_{p}(X) \times C_{p}(Y)$ is Lindelöf.

Theorem 4.1.5. (PFA) Let $X$ be a space with a single non-isolated point. The following properties are equivalent:

1. $\quad X$ is Lindelöf and $t(X)$ is countable.

2. $C_{p}(X)$ is Lindelöf.

\section{2 $X$ is a Michael Space}

The following specific case that we will examine considers a space $X$ which is a Michael space.

Definition 4.2.1. Let $X$ be a topological space and $A \subseteq X$. Let $X_{(A)}$ be obtained by retaining the topology at each point of $A$ and by taking the points of $X \backslash A$ isolated. Then $X_{(A)}$ is referred to as a Michael space.

The following are a few properties of Michael spaces that are required before considering questions of Lindelöfness in $C_{p}(X)$.

Proposition 4.2.2. Let $A$ and $B$ be two subsets of a topological space $X$. Then, there exists a one-to-one mapping $i: X_{(A)} \rightarrow X_{(B)}$, where $i$ is the identity mapping on $X$. If $A \subseteq B$, this mapping is continuous.

Proposition 4.2.3. The diagonal in $X_{(A)} \times X_{(B)}$ is closed and homeomorphic to $X_{(A \cap B)}$.

The following definitions are necessary to understand the findings for Michael spaces. 
Definition 4.2.4. Let $X$ be a topological space and $A \subseteq X$. Then, $A$ is a holding if the countable power $\left(X_{(A)}\right)^{\omega}$ is Lindelöf.

Definition 4.2.5. A separable completely metrizable topological space is called a Polish space. Equivalently, a Polish space is a topological space which is homeomorphic to a complete metric space with a countable dense subset.

In [7], Okunev and Tamano proved the following:

Theorem 4.2.6. If $A$ is a set in a Polish space $X$ such that $|X \backslash A|<2^{\omega}$, then $A$ is a holding.

Theorem 4.2.7. Let $X$ be an uncountable Polish space. Then there exists a family of pairwise disjoint holding subsets $\left\{A_{\alpha}: \alpha<2^{\omega}\right\}$.

The constructions of the previous two theorems would lead Okunev and Tamano to the following results:

Theorem 4.2.8. There exist separable, scattered, $\sigma$-compact spaces $X$ and $Y$ such that $C_{p}(X)^{\omega}$ and $C_{p}(Y)^{\omega}$ are Lindelöf but the product $C_{p}(X) \times C_{p}(Y)$ is not Lindelöf.

Theorem 4.2.9. (CH) Let $D=\{0,1\}$ denote the discrete space consisting of two points. There is a separable, scattered, $\sigma$-compact space $X$ such that for any finite $n$ $C_{p}(X, D)^{n}$ is Lindelöf, but $C_{p}(X, D)^{\omega}$ is not Lindelöf.

\section{3 $X$ is a Mrówka Space}

The final example we will look at in this chapter revolves around $C_{p}(X)$ when $X$ is a Mrówka space. To begin, we recall the following definitions.

Definition 4.3.1. Two countable infinite sets are said to be almost disjoint if their intersection is finite.

Definition 4.3.2. A maximal almost disjoint family is a maximal element in the family of all almost disjoint families ordered by inclusion. 
With the previous definitions in mind, we are able to define a Mrówka space.

Definition 4.3.3. Let $\mathcal{A}$ be a maximal family of pairwise, almost disjoint, infinite subsets of $\mathbb{N}$. We place a topology on the union $\mathbb{N} \cup \mathcal{A}$ by letting sets of the form

$$
U_{n}(A)=(A \backslash\{1,2, \ldots, n\}) \cup\{A\}, \quad n \in \mathbb{N},
$$

be basic neighborhoods of $A \in \mathcal{A}$ and declaring points of $\mathbb{N}$ to be isolated. The resulting space $\mathbb{N} \cup \mathcal{A}$ is called a $\Psi$-space or Mrówka space and is denoted $\Psi(\mathcal{A})$.

Some properties of the space $X=\Psi(\mathcal{A})$ are considered below.

Proposition 4.3.4. For every almost disjoint family $\mathcal{A}$, the space $X=\Psi(\mathcal{A})$ is a zero-dimensional, locally compact, first countable space. Additionally, $\mathcal{A}$ is a closed discrete subspace of $\Psi(A)$ and $\omega$ is dense.

Theorem 4.3.5. $\Psi(\mathcal{A})$ is not normal if $\mathcal{A}$ is a maximal almost disjoint family.

An important result with Mrówka spaces was proven in a 2005 paper by Hrusak, Szeptycki and Tamariz- Mascarua [3]. The following theorem is stated assuming CH.

Theorem 4.3.6. There is a maximal almost disjoint family $\mathcal{A}$ such that for $X=$ $\Psi(\mathcal{A})$ the finite power $C_{p}(X, D)^{n}$ is Lindelöf for every finite $n$, but the countable product $C_{p}(X, D)^{\omega}$ is not normal. 


\section{Chapter 5}

\section{$C_{p}(X)$ and Metacompactness}

In this chapter we will look at the spaces $C_{p}(X)$ and the property of metacompactness. Several open questions on the topic are presented in this section. However, despite the numerous questions presented on this subject, there are very few answers available to date. We begin with some of these unanswered questions.

Question. Does metacompactness of $C_{p}(X)$ imply that $C_{p}(X)$ is Lindelöf?

This question, first asked by Arhangel'skii in [1], is often considered to be a part of $C_{p}$-theory folklore. While we do not have a complete answer, there is a potential one due to a theorem by Reznichenko [2]. First we begin with a definition.

Definition 5.0.7. A space $X$ is called collectionwise normal if, for any discrete $\left\{F_{t}: t \in T\right\}$ of closed subsets of $X$, there exists a discrete family $\left\{U_{t}: t \in T\right\}$ of open subsets of $X$ such that $F_{t} \subseteq U_{t}$ for each $t \in T$.

Theorem 5.0.8. If the space $C_{p}(X)$ is normal, then it is collectionwise normal.

This previous theorem, gives an important result [1]:

Theorem 5.0.9. If $C_{p}(X)$ is normal and metacompact, then $C_{p}(X)$ is Lindelöf.

The next question follows naturally from the above, and questions the necessity of $C_{p}(X)$ being normal [10].

Question. (open reference) Suppose that $C_{p}(X)$ is metacompact. Must it be Lindelöf? 
In a contradiction to the conjecture from [2] stated at the end of Chapter 3, Tkachuk in [11] believes that there are counterexamples that exist to that conjecture. He does however, believe that the Lindelöf property of $C_{p}(X)$ effects $C_{p}(X) \times C_{p}(X)$ as illustrated in the question below.

Question. Suppose that $C_{p}(X)$ is Lindelöf. Must $C_{p}(X) \times C_{p}(X)$ be metacompact?

The following question naturally follows from the preceding question, but first we must define metalindelöf [10].

Definition 5.0.10. A space $X$ is metalindelöf if every open cover $\mathcal{U}$ of $X$ has a point-countable open refinement.

Question. Suppose $C_{p}(X)$ is Lindelöf. Must $C_{p}(X) \times C_{p}(X)$ be metalindelöf?

Finally, we end with an open question that involves putting more constraints on the space $X[10]$.

Question. Suppose that $X$ is compact $C_{p}(X)$ is Lindelöf. Must the space $C_{p}(X) \times$ $C_{p}(X)$ be metacompact? 


\section{References}

[1] A.V. Arhangel'skii. Topological Function Spaces. Kluwer Academic Publishers, Boston, 1992.

[2] A.V. Arhangel'skii. Embeddings in $C_{p}$-spaces. Topology and its Applications, 85:9-33, 1998.

[3] M. Hrušák, P.J. Szeptycki, and A. Tamariz-Mascarua. Spaces of continuous functions defined on Mrówka spaces. Topology and its Applications, 158(1-3):239$252,2005$.

[4] A.G. Leiderman and V.I. Malykhin. Final compactness is not preserved under multiplication of spaces of the form $C_{p}(X)$. Siberian Mathematical Journal, 29(1):65-72, January 1988.

[5] Arkady Leiderman. On Lindelöf $C_{p}(X)$ spaces. Ben-Gurion University of the Negev, February 2007.

[6] O.G. Okunev. On Lindelöf sets of continuous functions. Topology and its Applications, 63:91-96, 1995.

[7] Oleg Okunev and Kenichi Tamano. Lindelöf powers and products of function spaces. Proceedings of the American Mathematical Society, 124(9):2905-2916, 1996.

[8] M.G. Tkachenko and V.V. Tkachuk. Dyadicity index and metrizability of compact continuous images of function spaces. Topology and its Applications, 149:243-257, 2005.

[9] V. V. Tkachuk. A $C_{p}-$ Theory Problem Book: Topological and Function Spaces. Springer, New York, 2011.

[10] V.V. Tkachuk. Open Problems in Topology II, chapter Twenty questions on metacompactness in function spaces, pages 595-598. Elsevier, New York, 2007.

[11] V.V. Tkachuk. A selection of recent results and problems in $C_{p}$-theory. Topology and its Applications, 154:2465-2493, 2007.

[12] A. Tychonoff. Über einen Funktionenräum. Mathematische Annalen, 111:762$766,1935$. 
[13] S. Willard. General Topology. Dover, New York, 2004. 